\title{
RNA interference: recent trends and its application in controlling Neglected Tropical Diseases (NTDs)
}

\author{
Alpha KARGBO ${ }^{1,4^{*}}$, Edrissa JAWO ${ }^{1}$, Pierre A. MENDY ${ }^{1,2}$ and Moses Edache ENTONU ${ }^{3}$ \\ ${ }^{I}$ Department of Physical and Natural Sciences, University of The Gambia. \\ ${ }^{2}$ Department of Biosciences, COMSATS University Islamabad, Pakistan. \\ ${ }^{3}$ Department of Biochemistry, Ahmadu Bello University, Zaria, Nigeria. \\ ${ }^{4}$ WASCAL-GRP, University Felix Houphouet Boigny, Ivory Coast. \\ ${ }^{*}$ Corresponding author; E-mail: akargbo@utg.edu.gm
}

\begin{abstract}
Degradation of homologous mRNA in some cells or organisms is possible through the introduction of double-stranded RNA (dsRNA), via a process called RNA interference (RNA $i$ ). The dsRNAs are processed into short interfering RNAs (siRNAs) which consequently bind to the RNA-induced silencing complex (RISC), and causes degradation of target mRNAs. RNA $i$ has been widely used to study gene modifications and functions hence has the possibility to control disease pathogens or vectors. This promising potential led researchers to discovery gene control mechanisms in tropical diseases, by manipulating genes of pathogens and vectors, protozoans, animal parasitic helminthes and disease-transmitting vectors, such as insects. Many pathogens and vectors cause severe parasitic diseases in tropical regions and it is challenging to control them once the host has been invaded intracellular. The aim of this work is to show how RNA interference can be used as treatment candidate for controlling some neglected tropical disease as it is highly effective in impeding parasitic development and their proliferation within their host.
\end{abstract}

(C) 2020 International Formulae Group. All rights reserved.

Keywords: Mosquitoes, Parasitic protozoa, Helminthes, Insect vector.

\section{INTRODUCTION}

Hundreds of organisms have had their genomes sequenced and this holds the potential of revealing the gene functions in most of these organisms. The idea of genome sequencing has aided researchers to progress more comprehensive methods to understanding the biology of living organisms (Liolios et al., 2006). However, functions of over half of the genes in sequenced species remained unknown, needing annotation to fully appreciate the biological functions of those genes. RNA interference (RNA $i$ ) has been extensively used as a molecular tool by which target transcripts can be cut, resulting in the reduction of mRNAs for protein expression (Novina and Sharp, 2004). Even though other genetic modifications such as Germ line transformation modify the genotypes of target organisms, introduction of double-stranded RNA (dsRNA) changes only the phenotypes of organisms without modifying the genotypes (Kang and Hong, 2008). The dsRNA reduces the transcripts of specific mRNA, instead of deleting or inserting a gene like genetic modifications. The RNA $i$ method has been 
regularly used for analyzing gene function and has the potential to be a tool for disease therapy by interfering with vector competence or pathogen development (Shuey et al., 2002). This indication has been a focal point for controlling tropical diseases in recent times. The designation "Tropical Diseases" has been part of the medical vocabulary since the 19th century. On the "Tropical Diseases" Web page of World Health Organization (WHO), a list of eight diseases that occurred exclusively in the tropics and for all practical purposes, the term "Tropical Diseases" refers to infectious diseases that proliferate in hot and humid weather conditions. Some of these diseases are caused by protozoa, which include; malaria, leishmaniasis, Chagas' disease and sleeping sickness. Others are caused by worms, including; Schistosomiasis, Onchocerciasis and lymphatic Filariasis. One is viral, identified as dengue fever. The eight WHO Tropical Diseases are spread to humans by various means, but always a vector is involved and is generally a haematophagous insect. Schistosomiasis has no vector, but rather intermediary hosts - snails - that discharge in water the infectious forms for humans (Camargo, 2008). Most of these tropical diseases are neglected, including six other parasitic and bacterial diseases were recognized as being neglected but are found to be among some of the most common diseases that cause infections in the projected that, 2.7 billion people who live on less than $\$ 2$ per day. These diseases occur principally in rural areas and in some poor urban settings of low-income countries in sub-Saharan Africa, Asia, and Latin America (Camargo, 2008).

These thirteen (13) parasitic and bacterial infections are documented as "Neglected Tropical Diseases" and they comprise three soil-transmitted helminthic infections (ascariasis, hookworm infection, and trichuriasis), lymphatic filariasis, onchocerciasis, dracunculiasis, schistosomiasis, Chagas' disease, human African trypanosomiasis, leishmaniasis, Buruli ulcer, leprosy, and trachoma (Molyneux et al., 2005; Hotez et al., 2006). An extended list could include dengue fever, the treponematoses,

leptospirosis, strongyloidiasis, foodborne trematodiases, neurocysticercosis, and scabies, (Hotez et al., 2006) as well as other tropical infections. The devastating effects of these tropical diseases had called for much importance on their control. Although monitoring measures involving environmental sanitation, observation, early diagnosis and treatment and vector control (Fevre et al., 2006; Yamagata and Nakagawa, 2006; Alvar et al., 2006). RNA $i$ seems encouraging in silencing gene expression in some of these parasitic pathogens such as protozoans and helminthes, as well as disease vectors by exactly targeting mRNA interference. Investigation of gene functions in pathogens of infectious diseases and their vectors is significant for research in drug development, and the silencing effects may be directly employed to regulate parasite transmission and development (Kang and Hong, 2008). Nevertheless, there are certain practical obstacles when using RNA $i$ in tropical disease pathogens. RNA $i$ technology could have been eliminated in certain parasitic protozoans, hence the existence of RNA $i$ pathway should be established before utilization (Ullu et al., 2004). While using RNA $i$, the necessities for an ideal delivery method should be recognized to generate optimal RNA $i$ effects. The dsRNA-generating vector systems can be employed to explain the issue of transient RNA $i$ effects. It is also used in the treatment of several forms of diseases such as cancer, heritable disease etc. Due to difficulty to treat genetic diseases and also control animal and plant pest in recent times, the mechanism of RNA interference is employed to target specific pathogen or disease. The use of RNAi is an example of several biotechnological tools which can be used to manipulate the genes of organism in order to solve problems faced by man (Kargbo et al., 2020). The aim of this work is to show how RNA interference can be used as treatment candidate for controlling some neglected tropical disease as it is highly effective in impeding parasitic development and their proliferation within their host. 


\section{RNA $i$ IN THE CONTROL OF TROPICAL DISEASES BY PARASITIC HELMINTHS}

The control of tropical diseases initiated by parasitic helminths has engaged the contributions of RNA $i$. Silencing characteristic of RNA $i$ to helminths genes may kill the worms directly or interferes with important functions necessary for their development. It can also be used as a tool to study gene functions in helminths, just as it has been used in other species. Moreover, genes recognized by RNA $i$ can be useful as target genes for drug development or vaccine candidates, allowing utilization of RNA $i$ for therapeutic purposes (Kang and Hong, 2008). To date, only 10 species ( 8 in nematodes and 2 in trematodes) have been studied for RNA $i$ effects in animal parasitic helminths (Geldhof et al., 2007). This is somewhat surprising considering the fact that the nematode, Caenorhabditis elegans was the first organism in which dsRNAs were proven to be an RNA $i$ inducing factor and it has served as a model organism to describe the RNA $i$ mechanism (Kang and Hong, 2008). Below are some of the species of parasitic helminths in which RNA $i$ has been studied.

\section{Nematodes}

\section{Nippostrongylus brasiliensis}

Nippostrongylus brasiliensis, is a rat intestinal parasitic nematode, this parasite is an important animal model since it shares an analogous life cycle with the human hookworms, Necator americanus and Ancylostoma duodenale. When 1,799bp-long dsRNAs targeting full length of acetylcholinesterases (AchEs) cDNA at the concentration of $1 \mathrm{mg} / \mathrm{ml}$ dsRNA were employed, the target gene was blocked nearly by $80 \%$ on the first day, but then the transcripts returned to normal levels in 4 days later. However, by targeting AchEs with 240bp of dsRNAs, AchEs were blocked by more than $90 \%$ and the effects lasted for 6 days, signifying that the short dsRNA were effective in suppressing the target gene expression (Hussien et al., 2002). This experiment provided the first confirmation of a successful RNA $i$ effect in parasitic helminthes. Therefore, control of helminths via RNA $i$ appears to be feasible once a suitable target gene is recognized (Kang and Hong, 2008).

\section{Brugia malayi}

RNA $i$ effects in Brugia malayi showed a more promising control method while targeting housekeeping genes ( $\beta$-tubulin and RNA polymerase II large subunit) (Aboobaker and Blaxter, 2003). This study showed that $300 \mathrm{bp}$ long dsRNA was very effective enough to result in the death of the filarial worm. The authors also utilized RNA $i$ to target another gene, microfilaria sheath protein $1 / \mathrm{mf} 22$, but this was not lethal to the worms although microfilariae release was abridged and half of the released microfilariae did not have fully elongated sheathes. B. malayi is an important lymphatic filarial nematode and it is challenging to block their transmission by mosquitoes. Therefore, the lethal effect from RNA $i$ was substantial as it proved to be a latent control system for B. malayi. Although earlier studies have reported a high concentration of dsRNA was necessary to knock down target genes in various helminths (Hussein et al., 2002; Aboobaker and Blaxter, 2003; Cheng et al., 2005). Pfarr et al., (2006) claimed that low concentration of dsRNA was enough to definitely knock down a target gene in Litomosoides sigmodontis, a rodent filaria. All concentrations ranging from 0.035 to 35 Mof dsRNA comparably reduced actin gene transcripts in adult worms by more than $90 \%$. They also measured induction of hsp60 gene to find out any stressful reaction on dsRNA injection. These concentrations of Mof dsRNAs reduced the target with the least difference and no hsp60 induction was observed, but high concentrations (17.5 and $35 \mathrm{M}$ ) resulted in a significant increase in hsp60 transcripts, indicating that a stress level in the filarial worm by high dsRNA dosage. Their study however, recommends that low concentrations are enough to reduce transcript levels steadily whereas high concentrations of dsRNA may be stressful to the filarial worms. Thus, titration of appropriate concentration of dsRNAs would be required prior to RNA $i$ experiments (Kang and Hong, 2008). 


\section{Onchocerca volvulus}

Functional RNA $i$-knockdown was described in L3 larvae of Onchocerca volvulus. Lustigman et al. (2004) targeted cathepsin L and cathepsin Z-like cysteine proteases that showed a vital role in L3 to L4 larvae for molting. Soaking the third-stage larvae (L3) in a dsRNA solution reduced the molting rate by $92 \%$ for cathepsin $\mathrm{L}$ and $86 \%$ for cathepsin Zlike cysteine proteases. Gene silencing of these cathepsin transcripts hindered the molting process by 1-3 days, this shows a significant reduction in the viability of the L3 larvae in $O$. volvulus (Ford et al., 2005). Gene silencing of inorganic pyro phosphatase of the parasitic round worm, Ascaris suum, prevented molting from L3 to L4 by 31\% (Islam et al., 2005). Although the inhibition rate was inferior than that of $O$. volvulus, discovery of functional gene silencing by RNA $i$ in Ascaris worms was significant and this can be further exploited as a model system to study RNA $i$ in human ascariasis (Kang and Hong, 2008). Issa et al., (2005) recommended that siRNA and electroporation are more efficient molecules and a delivery method, respectively, to induce gene silencing by RNA $i$ in the sheep gastrointestinal parasite, Trichostrongylus colubriformis. The authors tested three different RNA $i$ delivery methods; feeding of Escherichia coli expressing dsRNA, soaking of siRNA or dsRNA, and electroporation of siRNA or dsRNA. Ubiquitin and tropomyosin were used as target genes since their DNA sequences are well conserved and readily available. Ubiquitin transcripts were not reduced by the E. coli feeding method, but tropomyosin was suppressed. siRNA in both electroporation and soaking resulted in a significant reduction for both target genes (Kang and Hong, 2008). Their study showed that it is likely to have electroporation which will result in more reliable gene silencing, and the 22bp siRNA will be more effective than long dsRNA in reducing expression of both target genes.

\section{Haemonchus contortus}

A diversity of RNA $i$ conditions were tested to optimize RNA $i$ effects in Haemonchus contortus, a barberpole worm.
Various life stages (L1- L4 and adult), 11 genes ( $\beta$-tubulin, sec-23, $\mathrm{Ca}^{2+}$ binding protien, HSP70, vacuolar ATPase, cathepsin L, paramyosin, $\mathrm{Cu}-\mathrm{Zn}$ superoxide dismutase, intermediate filament, type IV collage and GATA transcription factor) and 3 different RNA $i$ delivery methods (feeding, soaking and electroporation) were tested in this organism to investigate RNA $i$ (Geldhof et al., 2006; Kotze and Bagnall, 2006). Two -tubulin genes that were targeted by RNA $i$ affected 3 life stages (L3, L4 and adult) and reduced target gene transcripts by the soaking method, still reduced motility and viability were only shown in the L3 stage (Kotze and Bagnall, 2006). Geldhof et al., (2006) tested RNA $i$ effects on 11 different genes of the L1-L3 stages by 3 different delivery methods in $H$. contortus. The feeding method was not effective in reducing target gene transcripts, confirming previous data in Trichostrongylus (Issa et al., 2005). Only two transcripts ( $\beta$-tubulin and sec-23) out of 11 genes in the L3 stage were significantly reduced by soaking in dsRNA. Fascinatingly, no phenotypic change was observed in the L3 larvae soaked in siRNA, and some control siRNA was even toxic to the L1 / L2 larvae (Kang and Hong, 2008). Electroporation was effective in reducing target gene transcripts in L1 larvae, as transcript levels of $\beta$-tubulin and superoxide dismutase were significantly decreased. However, larval death was observed in the L1/L2 stage by electroporation even in the presence of control dsRNA, signifying either electroporation is not a stable delivery method to this stage or current electroporation protocols for this stage are not optimal for analyzing RNA $i$ effects (Kang and Hong, 2008). Similarly, eight genes were tested in the L1 and L3 larval stages of Ostertagia ostertagi, a cattle parasitic nematode, by electroporation and soaking delivery methods. Substantial reduction of transcripts was detected for five target genes (tropomyosin, $\beta$-tubulin, ATPase, superoxide dismutase and a polyprotein allergen) in L3 larvae, but dsRNAs of a transthyretin-like protein, a $17 \mathrm{kDa}$ ES protein and ubiquitin did not reduce the target gene transcript levels (Kang and Hong, 2008). Electroporation was less effective, as only two 
genes (tropomyosin and $\beta$-tubulin) were effectively silenced, and these RNA $i$ effects were not even reproducible (Visser et al., 2006). This non-reproducible result indicated the RNA $i$ delivery method will require further optimization to realize consistent results (Kang and Hong, 2008).

\section{Trematodes}

Most RNA $i$ studies in trematodes mainly focused on Schistosoma spp, blood flukes, which cause hematemesis or liver failure, and may result in mortality.

\section{Schistosoma spp.}

Schistosoma worms causes a disease known as Schistosomiasis. It is one of the disease transmitted by freshwater snails. It affects about 200 million people in the world and about 600 people are at risk of infection in Africa, Latin America and Asia (Ntonifor and Ajayi, 2007). The first report of RNA $i$ in Schistosoma targeted the cathepsin B gene (Skelly et al., 2003), an enzyme previously proposed to be responsible for degradation of host hemoglobin to digestible peptides (Brindley et al., 1997). Schistosomes were soaked in dsRNA targeting cathepsin B and cultured for 6 days. The authors confirmed by RT-PCR that parasites soaked in the dsRNAs showed reduced target gene expression. Subsequent studies recommended electroporation as an alternative way to introduce dsRNA, and reduction of transcript levels of cathepsin B in schistosomula was established. Later RNA $i$ research showed that cathepsin B is needed for parasite growth, and not crucial for hemoglobin digestion (Correnti, et al., 2005). A much recent research was also conducted to optimize the application of RNAi to Genomic drug target validation in Schistosomes (Guidi, et al., 2015). The authors reported that there is no vaccine against the parasitoid, and the only potential cure relies on the use of the one available drug, praziquante this drug has been confirmed to be safe and effective against adult worms in a single dose administration (Zongo et al., 2016). However, the growing threat of emerging resistance makes the search for novel drugs a compelling priority. They reported that S. mansoni possesses a quite huge and complex genome so new in silico strategies to select genes which might represent possible novel drug target candidates and could be an important approach to drug development.

In their study, an initial list of 24 target candidates was compiled based on the identification of putative essential genes in Schistosoma orthologous of C. elegans essential genes. Knockdown of Calmodulin (Smp_026560.2) (Sm-Calm), topped the list, which produced a phenotype characterized by waves of contraction in adult worms but no phenotype in schistosomula. Knockdown of a typical Protein Kinase C (Smp_096310) (SmaPKC) resulted in the loss of viability in both schistosomula and adults. This had shifted their attention to other kinase genes that were identified in the above list and through whole organism screening of known kinase inhibitor sets followed by chemogenomic evaluation. RNAi knockdown of these kinase genes failed to affect adult worm viability but, like SmaPKC, knockdown of Polo-like kinase 1, SmPLK1 (Smp_009600) and p38-MAPK, SmMAPK p38 (Smp_133020) resulted in an increased mortality of schistosomula after 2-3 weeks, an effect additional marked in the presence of human red blood cells (hRBC). For Sm-PLK-1 the same effects were seen with the precise inhibitor, BI2536, which also affected viable egg production in adult worms. For $\mathrm{Sm}-$ PLK-1 and Sm-aPKC the in vitro effects were reflected in lower recoveries in vivo. They concluded that the use of RNAi combined with culture and hRBC is a reliable method for evaluating genes significance for larval development. However, in view of the slow manifestation of the effects of Sm-aPKC knockdown in adults and the lack of effects of Sm-PLK-1 and Sm-MAPK p38 on adult viability, they recommended that the kinases may not represent suitable drug targets. RNA $i$ was used to test another gene function in the sporocyst developmental stage of schistosomes. Boyle et al., (2003) knocked down SGTP1, a facilitated diffusion glucose transporter and glyceraldehyde-3-phosphate dehydrogenase (GAPDH) by RNA $i$. The expression of both genes in the sporocyst stage 
was reduced when miracidia, a free livinglarval stage of schistosome were soaked in its homologous dsRNA and allowed to transform to the sporocyst stage. Glucose transport activity was reduced when SGTP1 was knocked down, representing the function of the gene in schistosome. Fascinatingly, however, when dsRNAs were introduced at the sporocyst stage, the reduction was not observed, suggesting RNA $i$ is not effective when introduced into this developmental stage. The effect of RNA $i$ lasted for 28 days, which is similar to C. elegans (Tabara et al., 1998). RNA $i$ can, therefore, be introduced at the miracidia stage and the effects will last well into the sporocyst stage. RNA $i$ was used to reduce transcripts of scavenger receptors that are known to be substantial for the binding of low-density lipoprotein to the surface of schistosomula and adult S. mansoni. Because the parasites cannot produce sterols and fatty acids, uptake of host-lipoprotein by scavenger receptors is perilous in the synthesis of biological membranes (Kang and Hong, 2008). Miracidia were soaked in dsRNA targeting the scavenger receptors for 6 days and this resulted in a $60-70 \%$ reduction of the target gene transcript level. Decreasing of the scavenger receptors inhibited regular parasite development, showing a more rounded morphology in sporocysts and a shorter length in larval size (Dinguirard and Yoshino 2006). RNA $i$ was also used to target the gynecophoral canal protein $\mathrm{SjGCP}$, in Schistosoma japonicum, a major human pathogen in the Far East (Cheng et al., 2005). Schistosomes cannot develop without precise signaling during sexual pairing (Gupta and Basch et al., 1987), so disturbance of pairing would be good target in order to control schistosomes. It is known that $S$. mansoni GCP, an ortholog of SjGCP, is related to pairing and produced only by males (Bostic and Strand, 1996; Hoffmann, 2004). Cheng et al. (2005) utilized RNA $i$ to demonstrate that the SjGCP can be silenced in a dosage dependent style in $S$. japonicum. The target gene transcripts were reduced by $75 \%$ with $100 \mathrm{nM}$ of dsRNA, but not affected at $12.5 \mathrm{nM}$. This study recommended a potential therapeutic application of RNA $i$ in $S$. japonicum. With reduced $\mathrm{SjGCP}$, the mating in $S$. japonicum may be disturbed, which will cause inhibition of parasite development in the host.

\section{Fasciola spp.}

Fasciola spp. is a liver fluke that causes pernicious disease in humans and animals. Their present control is unmanageable due to anthelmintic resistance. Gene silencing (RNA interference, RNAi) has been shown to have potential to contribute to functional validation of new therapeutic targets. McVeigh et al. (2014), described that liver flukes reveal the persistence of gene silencing in-vitro assay showing the dynamics of RNAi in juvenile Fasciola spp. They described the development of standardized RNAi protocols for a commercially-available liver fluke strain (the US Pacific North West Wild Strain), validated via robust transcriptional silencing of seven virulence genes, with in-depth experimental optimization of three: cathepsin L (FheCatL) and $\mathrm{B}$ (FheCatB) cysteine proteases, and an sclass glutathione transferase (FhesGST). In their studies, the robust transcriptional silencing of targets in both $F$. hepatica and Fasciola gigantic juveniles is attainable following exposure to long (200-320nt) dsRNAs or $27 \mathrm{nt}$ short interfering siRNAs. They described a set of simple, soaking-based methods that permit rapid, robust and persistent knockdown of target transcripts, and address target-specific aspects of the time course of this RNAi response, which is mainly evident in the time lag between transcript and protein knockdown. The Knockdown was noticeable following as little as $4 \mathrm{~h}$ exposure to trigger (target-dependent) and in all cases silencing continued for $\geq 25$ days following long dsRNA exposure (Cheng et al., 2005). Combinatorial silencing of three targets by mixing multiple long dsRNAs was similarly efficient. Despite profound transcriptional suppression, they found a significant time-lag before the incidence of protein suppression; FhesGST and FheCatL protein suppression were only detectable after 9 and 21 days, respectively. They established that in spite of marked variations in knockdown dynamics, a transient exposure to long dsRNA or siRNA activates 
robust RNAi penetrance and persistence in liver fluke NEJs supporting the development of multiple-throughput phenotypic screens for control target authentication Cheng et al. (2005). According to McVeigh et al. (2014), RNAi persistence in fluke encourages in vivo studies on gene function using worms exposed to RNAi-triggers prior to infection.

\section{CURRENT CHALLENGES AND PROSPECTS OF RNA $i$ IN HELMINTHS}

Though genes are successfully silenced by RNA $i$ in $C$. elegans, gene knockdown by RNA $i$ has been either impossible or inconsistent in other helminths (Geldhof et al., 2007). For instance, Geldhof et al. (2006) tested efficacy of RNA $i$ silencing in the strong lid parasitic nematode, $H$. contortus, using three different dsRNA delivery methods; feeding, soaking, or electroporation. In this study, no RNA $i$ was observed with dsRNA feeding among four genes tested and only two genes out of eleven, beta-tubulin and sec-23, showed specific gene silencing by the soaking method. Similarly, two genes out of four had knockdown of target transcripts by electroporation of respective dsRNAs. Moreover, extents of gene silencing by RNA $i$ also varied in the cattle parasitic helminths, $O$. ostertagi and the results were often challenging to reproduce (Visser et al., 2006). Disagreement of results of RNA $i$ in parasitic helminthes may be due to different delivery methods employed for introducing dsRNAs into helminthes (Geldhof et al., 2006). There seems to be no consensus as to which is the most efficient delivery method to induce RNA $i$ gene silencing in parasitic helminths. Numerous reports have showed electroporation was an efficient alternative to the soaking method in delivering dsRNA in $T$. colubriformis and schistosomes (Issa et al., 2005; Correnti et al., 2005; Krautz et al., 2007). In contrast, others suggested that electroporation was not effective in $O$. ostertagi and in fact lethal to certain stages of H. contortus (Geldhof et al., 2006; Visser et al., 2006). Feeding dsRNA is largely inefficient in reducing transcript levels of target genes except for tropomyosin in T. colubriformis
(Issa et al., 2005). Soaking is another popular method for gene silencing, but this method was less efficient for the L1 / L2 stage of $H$. contortus (Geldhof et al., 2006) and for $S$. mansoni (Krautz et al., 2007). Apparently, all major methods normally used for RNA $i$ in $C$. elegans have their limitations in the delivery of dsRNA into parasitic helminths. The disagreement in RNA $i$ efficiencies among parasitic helminths may result from the absence of sid (systemic RNA interferencedeficient)- 1 , sid-2 or $r s d$ (RNA $i$ spreading defective)-4 in some of parasitic helminths. SID-1, SID-2, and RSD-4 proteins are involved in cellular uptake and spread of dsRNA in $C$. elegans (Viney and Thompson, 2008). SID-1 is a trans-membrane protein that is required for RNA $i$ uptake into cells and spread between cells. Therefore, the lack of these proteins in certain helminths can be stopped externally, preventing dsRNA from entering cells, making worms refractory to RNA $i$. In sid- 1 mutant $C$. elegans, systemic spread of siRNA molecules was defective with any dsRNA delivery methods (feeding, soaking and microinjection) (Winston et al., 2002). SID-2 is also a transmembrane protein, of which expression is incomplete in the apical membrane of the intestinal lumen. Therefore, SID-2 is believed to be essential for dsRNA uptake from the lumen to cells but not for spreading siRNA between cells (Kang and Hong, 2008). In sid-2 deficient $C$. elegans resistant to RNA $i$ by soaking and feeding methods, dsRNA delivered to the pseudo-coelom (body cavity) by microinjection could initiate RNA $i$ and siRNA that could independently spread into cells (Winston et al., 2007). As a result, sid-2 deficient $C$. elegans became vulnerable to RNA $i$, showing reduction of target transcripts. Likewise, $r s d-4$ mutant $C$. elegans resistant to RNA $i$ by feeding was reverted to be susceptible to RNA $i$ by supplying external dsRNA into the pseudocoelom using microinjection (Tijsterman et al., 2004). Therefore, it seems there is functional overlapping between sid-2 and $r s d-4$ proteins for systemic spread of siRNA (Kang and Hong, 2008). Many parasitic animal nematodes are deficient of sid-1, sid-2, or $r s d-4$ orthologs, or their homologs do not 
share the same functions with their counterparts in C. elegans (Viney and Thompson, 2008; Winston et al., 2002; Tijesterman et al., 2004). The lack of sequence or functional conservation of sid-1, sid-2, or $r s d-4$ may account for the discrepant RNA $i$ results between $C$. elegans and animal parasitic nematodes. To oppose this explanation, despite the fact that there is no identifiable sid-1 ortholog in B. malayi, a functional RNA $i$ mechanism was detected by a soaking method (Aboobaker and Blaxter, 2003). This suggested, that there may be another molecules or pathways substituting sid-1 for systemic spread of siRNAs in B. malayi. Therefore, it remains to be seen how systemic RNA $i$ was achieved without sid-1 orthologs in B. malayi (Kang and Hong, 2008). In order to have a phenotypic knock-down of specific gene for functional studies and therapeutic applications, definite modifications or enhancements for the RNA $i$ delivery methods may be obligatory in animal parasitic helminths. To this end, two approaches have been recently suggested (Viney and Thompson, 2008).

$>$ Firstly, one may be able to trigger RNA $i$ by microinjection of dsRNA into the pseudocoelom of parasitic helminths if failure of RNA $i$ by soaking or feeding is due to the absence of sid-2 orthologs in the gut lumen. This modification is based on the observation in Caenorhabditis briggsae, in which unlike $C$. elegans there is no sid-2 ortholog functionally conserved to allow entry of dsRNA from the gut lumen to the body cavity. Therefore, RNA $i$ cannot be initiated in $C$. briggsae by dsRNA using soaking and feeding methods. However, RNA $i$ was possible when dsRNA was supplied to the pseudocoelom via microinjection, suggesting that microinjection can bypass barriers of dsRNA uptake in the lumen, making RNA $i$ possible in $C$. briggsae (Winston et al., 2002; Baird and Chamberlin, 2006).

$>$ Secondly, RNA $i$ may be feasible in sid-1 or 2-deficient parasitic helminths by heterologous expression of sid-1 and / or sid-2 of C. elegans as shown by Winston et al., 2002.

In C. briggsae, for example, there is no functionally conserved sid-2 and RNA $i$ by soaking or feeding is thus impossible. However, transformation of $C$. briggsae with C. elegans sid-2 allowed the uptake of dsRNA from the lumen in soaking experiments, generating systemic RNA $i$ in $C$. briggsae (Winston et al., 2002). As transgenesis of helminths becomes available, development of an RNA $i$ system through heterologous expression of C. elegans genes for soaking or feeding may have promising applications for gene functional studies and target identification for drug discovery (Kang and Hong, 2008). $\mathrm{RNA} i$ screen is a less challenging method for drug target studies in helminths compared to forward genetic research (Shuey et al., 2002). If, however, RNA $i$ machinery requires complicated dsRNA delivery process in animal parasitic nematodes, C. elegans can be served as a model system to study gene functions. Since RNA $i$ machinery is well characterized in C. elegans, this experimental system can provide a potent tool for unveiling gene functions in other nematodes via comparative genomics (Jones et al., 2005). The feasibility of this approach has been lately demonstrated in B. malayi (Kumar et al., 2007). Using sequence analyses and comparisons including gene functional studies with RNA $i$ knockdowns in C. elegans, 589 B. malayi genes were recognized as being critical to the survival of the filarial worm, representing potential targets for anti-filarial drug discovery. Interestingly, among those 589 genes, 10 out of top 40 candidates were previously known to be promising targets for the drug discovery because of their roles in molting, central metabolism, and structural components (Kang and Hong, 2008). This method may be realistic only for animal parasitic nematodes whose genomes are fully sequenced such as $B$. malayi (Ghedin et al., 2007). Thus, genome sequencing of significant helminths should be a prerequisite for this model animal based drug target studies. 


\section{RNA $i$ AND THE CONTROL OF TROPICAL DISEASES BY PARASITIC PROTOZOA}

\section{Trypanosomatids}

RNA $i$ in $T$. bruce $i$ has been studied comprehensively since the machinery was discovered in the organism (Ngo et al., 1998). $T$. brucei has been used to elucidate the functions of proteins comprising the RNA $i$ machinery such as Ago1 and dicer (Shi et al., 2004, 2006, 2007), as well as basic RNA $i$ mechanisms. Researchers constructed a stemloop that generated hairpin- loop dsRNAs targeting the -tubulin gene under the control of the tetracycline-inducible promoter. This system inhibited $\beta$-tubulin expression in $T$. brucei (Shi et al., 2000). (Wang et al., 2000) used a vector (pZJM) in which a PCR amplified gene fragment was ligated between opposing promoters to inhibit specific gene expression in T. brucei. Consequently, sense and antisense RNAs were concurrently synthesized and hybridized, producing dsRNAs molecules. The synthesis of dsRNA targeting $\beta$-tubulin transcripts was induced by tetracycline. It was confirmed that $T$. congolense, which is the causative agent of nagana disease in cattle, also has RNA $i$ machinery by utilizing a tetracycline-regulated vector expression system (Inoue et al., 2002). The constructed vector was transfected into $T$. congolense cells, inducing the same RNA $i$ mechanisms as in T. brucei. The -tubulin synthesis was reduced when the vector was transfected into a $T$. congolense cell line in the existence of tetracycline, demonstrating similar results to the earlier experiment targeting $\beta$-tubulin gene in $T$. brucei. Induction of the RNA $i$ pathway changed the morphology of transfected T. brucei cells after beta-tubulin transcripts were silenced (Ngo et al., 1998). This recommended that RNA $i$ machinery exists in T. congolense. Conversely, T. cruzi, Leishmania donovani and $L$. major lack RNA $i$ machinery although they belong to the same family as $T$. brucei that contains the required mechanisms of the RNA $i$ pathway (Zhang and Matlashewski, 2000; Robinson and Beverley, 2003; Darocha et al., 2004). Upon searching genome databases, it was found that these species are deficient in orthologs of the Ago1 protein. It is not surprising that RNA $i$ has not been observed without Ago1, since this protein is required for suppression of foreign and endogenous transgenes. Another missing component is the PAZ domain, generally present in Ago. The presence of a Piwi domain is indicative of a functional RNA $i$ mechanism because it is a part of the Ago protein that links with siRNA to cleave target mRNA (Saito et al., 2006). However, species that do not have an RNA $i$ mechanism lack proteins with a PAZ domain (Ullu et al., 2004), which is a subdomain of Ago and the dicer protein which binds to siRNA, contributing to firm incorporation of siRNA and miRNA into the RISC complex (Song et al., 2003). The deficiency of these domains may explain why certain species do not have an RNA $i$ pathway. A vector system has been studied in $T$. brucei and can be applied to different parasitic protozoan species to achieve temporal and spatial induction of RNA $i$. Some researchers described the role of antisense RNA in knocking down target mRNA in Plasmodium. Although this is not a conventional RNA $i$ pathway, it should be further exploited to use the antisense-knockdown system to silence specific genes (Noonpakedee et al., 2003).

\section{RNA $i$ IN INSECT VECTORS}

RNA $i$ has been used to silence target genes or investigate gene functions in many insect species. RNA $i$ induced by dsRNA was revealed in Drosophila both in vivo and in vitro (Kennerdell et al., 1998; Tuscgl et al., 1999). By eliminating engrailed (en) transcripts with RNA $i$, Marie et al. (2000) studied additional functions of the en gene that was previously known to control topography of axonal projections in D. melanogaster. Using larvae inserted with en dsRNA, a change in axonal branching and synaptic outputs was found, and thus demonstrating en controls synaptic choice as well as axonal projections. After fruitful trials of RNA $i$ in Drosophila, the RNA $i$ technique was applied to other insect species (Kang and Hong, 2008). 


\section{Tsetse fly}

Tsetse fly is the main vector for trypanosomes. The trypanosomes life cycle is an intermediate between the tsetse fly and its mammalian host (Ebene et al., 2016; Mamoudou et al., 2016) RNA $i$ was used to examine the role of a specific gene in tsetse flies (Nayduch and Aksoy, 2007). Tsetse flies, Glossina spp., transmit African trypanosomes which includes Africa Animal Trypanosomiasis and Human African Trypanosomiasis but the prevalence is low because the flies are refractory to trypanosome infection (Oyibo et al., 2009). Scientists have compared the transcription levels of an antimicrobial peptide gene attacin, between refractory G. palpalis palpalis and susceptible G. morsitans morsitans. The levels of attacin expression were higher in the refractory species, signifying that attacin may be involved in the refractoriness of G. palpalis palpalis. Subsequently, microinjection of dsRNAs targeting attacin was carried out in order to explain the role of the gene. When the attacin was silenced by dsRNAs, the infection rate meaningfully increased from $20 \%$ to $40 \%$. This study showed that attacin is a refractory gene against African trypanosomes and also demonstrated that RNA $i$ can be used as a powerful tool to examine gene functions in tsetse flies (Kang and Hong, 2008).

\section{Mosquito Species}

Mosquito species are commonly known to be vectors for malaria. The occurrence of malaria in Africa, together with the lack of functioning vaccine, and the occurrence of parasite resistance to conventional drugs makes it necessary to hunt for antimalarial drug or vaccine candidate for the treatment of malaria (Ngbolua et al., 2011). RNA $i$ can be employed to experiment a novel method for the control of this vector-borne diseases. Malaria control using germ-line transformation and RNA $i$ has been suggested (Osta et al., 2004). Malaria is prevalent in Sub-Saharan Africa and Southeast Asia where resources are limited for disease treatments and vector control. RNA $i$ exists in several mosquito species, many of which are important disease vectors (Kang and Hong,
2008). When a pre-membrane coding section of the dengue virus type 2 genome was expressed in C6 / 36 cells derived from Aedes albopictus in sense and antisense orientation, the titer of specific type 2 virus was declined, signifying that dsRNA can induce resistance to virus infection in mosquito cells (Gaines et al., 1996). The same resistance pathway against dengue virus type 2 was shown when the premembrane coding region of the virus was expressed in Aedes aegypti adult mosquitoes (Olson et al., 1996). These experiments clearly established the existence of RNA $i$ in Aedes species and suggested the possibility of the use of RNA $i$ to control vector-borne diseases. An. gambiae, a vibrant vector species for malaria, also has the RNA $i$ pathway (Blandin, et al., 2002). Injection of dsRNAs targeting the endogenous Defensin gene, an antimicrobacterial peptide gene, silenced the Defensin transcripts, which augmented the activity of gram-positive bacteria in $A n$. gambiae. This showed the possibility of RNA $i$ in elucidating gene functions in anopheline mosquitoes. Analysis of gene functions using RNA $i$ was employed to identify antiplasmodial genes in An. gambiae. Microarray analysis was performed in mosquitoes infected by $P$. falciparum or $P$. berghei, and 11 candidate immune genes (Tep1, AgMDL1, FBN8, FBN9, FBN39, SPCLIP1, APOD, IRSP1, IRSP5, $L R R D 7$, and gambicin) were identified, and the functions of these genes were assessed by RNA $i$ gene silencing to show their antiplasmodium activities. The dsRNAs of the 11 target genes were injected into mosquitoes and gene silencing was established by real-time RT-PCR. Silencing of each immune gene increased Plasmodium levels. However, insects do not share the same immune pathway. Thus, typical mammalian off-target effects may not cause concerns in insect vectors. Other common examples of off-target effects of RNA $i$ may be over-dose lethality and toxic effects (Geldhof et al., 2007). Soaking and electroporation are the main RNA $i$ delivery systems in protozoans and helminths, which may cause overall toxic effects due to long exposure time to dsRNA or injury by electroshock (Kang and Hong, 2008). Unlike 
parasitic pathogens, insect RNA $i$ delivery relies on mostly microinjection. Thus, offtarget effects caused by injection of nonphysiological amounts of dsRNAs may be preventable by careful titration of dsRNAs to be used for RNA $i$ experiments in insects (Kang and Hong, 2008).

\section{Conclusion}

The technique of RNA $i$ showed great potential in genetic manipulation and the development of therapeutic and control applications in many tropical disease pathogens and vectors. Its mechanism makes it efficient to analyze most gene functions in a way that reduces the gene expression without having to alter the genotypes. Hence, RNA $i$ gives us a better insight in understanding gene functions in parasites, find better drug targets and vaccine candidates, or reduce the vector competence to transmit most tropical diseases. Optimization of methods for delivering a specific RNA $i$ system into a particular organism gives the technique its success in application. Soaking and electroporation have been effectively used for the introduction of dsRNAs into parasites, and a microinjection protocol has been established to deliver dsRNAs into vector insects. These provide general guidelines for performing RNA $i$ gene silencing. However, there is need for modification of conditions for different gene knockdowns, in order to suit each system or organism since varying degrees of gene silencing have been observed among different target genes and delivery methods. The conventional RNA $i$ produces transient gene suppression effects; however, long-lasting effects may be necessary for permanent gene silencing. Germline transformation and a vector-based RNA $i$ system would be an answer to this transient knockdown issue. Factors that cause off-target effects need to be identified and avoided so as to ensure effective gene knockdown.

\section{COMPETING INTERESTS}

The authors declare that they have no competing interests.

\section{AUTHORS' CONTRIBUTIONS}

All authors contributed in writing and editing the manuscript.

\section{ACKNOWLEDGEMENTS}

The authors appreciate the contribution and support of all the P15 students of the Department of Biochemistry, School of Life Science, Ahmadu Bello University, Zaria, Nigeria.

\section{REFERENCES}

Aboobaker AA, Blaxter ML. 2003. Use of 2 RNA interference to investigate gene function in the human filarial nematode parasite Brugia malayi. Molecular Biochemistry Parasitology, 129: 41-51. DOI: https://doi.org/10.1016/s01666851(03)00092-6.

Alvar J, Croft S, Olliaro P. 2006. Chemotherapy in the treatment and control of leishmaniasis. Advance Parasitology, 61: 223-274. DOI: https://doi.org/10.1016/s0065-308x (05)61006-8.8

Baird SE, Chamberlin HM. 2006. Caenorhabditis briggsae methods. In: The C. elegans Research Community. WormBook.

DOI: 10.1895/wormbook.1.128.1, http://www.wormbook.org.

Blandin S, Moita LF, Kocher T, Wilm M, Kafatos FC, Levashina EA. 2002. Reverse genetics in the mosquito Anopheles gambiae: targeted disruption of the Defensin gene. EMBO Rep, 3: 852856.

Bostic JR, Strand M. 1996. Molecular cloning of a Schistosoma mansoni protein expressed in the gynecophoral canal of male worms. Molecular Biochemistry Parasitology, 79: 79-89. DOI: $\quad 10.1016 / 0166-$ 6851(96)02640-0.

Boyle JP, Wu XJ, Shoemaker CB, Yoshino TP. 2003. Using RNA interference to manipulate endogenous gene expression in Schistosoma mansoni sporocysts. Molecular Biochemistry Parasitology, 
128: 205-215. DOI: $10.1016 / \mathrm{s} 0166-$ 6851(03)00078-1.

Brindley PJ, Kalinna BH, Dalton JP, Day SR, Wong JY, Smythe ML, McManus DP. 1997. Proteolytic degradation of host hemoglobin by schistosomes. Molecular Biochemistry Parasitology, 89: 1-9. DOI: 10.1016/s0166-6851(97)00098-4

Camargo EP. 2008. Tropical Diseases. Estudos avançados, 22(64): 90-98. DOI: 10.1590/S0103-40142008000300008.

Cheng GF, Lin JJ, Shi Y, Jin YX, Fu ZQ, Jin YM, Zhou YC, Cai YM. 2005. Dosedependent inhibition of gynecophoral canal protein gene expression in vitro in the schistosome (Schistosoma japonicum) by RNA interference. Acta Biochemistry Biophysics, 37: 386-390. DOI: $10.1111 / \mathrm{j} .1745-$ 7270.2005.00058.x.

Correnti JM, Brindley PJ, Pearce EJ. 2005. Long-term suppression of cathepsin B levels by RNA interference retards schistosome growth. Molecular Biochemistry Parasitology, 143: 209215. DOI: 10.1016/j.molbiopara.2005.06.007.

DaRocha WD, Otsu K, Teixeira SMR, Donelson JE. 2004. Tests of cytoplasmic RNA interference (RNA $i$ ) and construction of a tetracycline-inducible T7 promoter system in Trypanosoma cruzi. Molecular Biochemistry Parasitology, 133: 175-186. DOI: 10.1016/j.molbiopara.2003.10.005.

Dinguirard N, Yoshino TP. 2006. Potential role of a CD36-like class B scavenger receptor in the binding of modified low-density lipoprotein (acLDL) to the tegumental surface of Schistosoma mansoni sporocysts. Molecular Biochemistry Parasitology, 146: 219-230. DOI: 10.1371/journal.pntd.0003185

Ebene NJ, Mingoas JP, Bayemi PH, Manchang TK, Mfopit MY, Onyali IO, Musongong GA Chiejina SN. 2016. Interaction between Trypanosoma brucei and Haemonchus contortus infection in West African Dwarf Goats. Int. J. Biol. Chem. Sci., 10(4): 1580-1589.
Ford L, Guiliano DB, Oksov Y, Debnath AK, Liu J, Williams SA, Blaxter ML, Lustigman S. 2005. Characterization of a novel filarial serine protease inhibitor, Ov-SPI-1, from Onchocerca volvulus, with potential multifunctional roles during development of the parasite. Journal Biology and Chemistry, 280: 40845-40856. DOI: 10.1074/jbc.m504434200.

Gaines PJ, Olson KE, Higgs S, Powers AM, Beaty BJ, Blair CD. 1996. Pathogenderived resistance to dengue type 2 virus in mosquito cells by expression of the premembrane coding region of the viral genome. Journal of Virology, 70: 21322137. DOI: $10.4269 /$ ajtmh.1998.58.663

Geldhof P, Murray L, Couthier A, Gilleard JS, McLauchlan G, Knox DP, Britton C. 2006. Testing the efficacy of RNA interference in Haemonchus contortus. International Journal of Parasitology, 36: $801-810 . \quad$ DOI: 10.1016/j.ijpara.2005.12.004

Geldhof P, Vissera A, Clarka D, Saundersa G, Brittona C, Gillearda J, Berrimana M, Knoxa D. 2007. RNA interference in parasitic helminths: current situation, potential pitfalls and future prospects. Parasitology, 134: 609-619. DOI: 10.1017/s0031182006002071.

Guidi A, Nuha RM, Ross AP, Ian MC, Jérémy B. Andrew LH, Ian HG, Quentin DB. 2015. Application of RNAi to Genomic Drug Target Validation in Schistosomes. PLoS Negleted Tropical Disease, 9(5): 3801.

DOI: 10.1371/journal.pntd.0003801

Gupta BC, Basch PF. 1987. Evidence for transfer of a glycoprotein from male to female Schistosoma mansoni during pairing. Journal of Parasitology, 73: 674675. DOI: $10.1006 /$ expr.1998.4343

Hoffmann KF. 2004. An historical and genomic view of schistosome conjugal biology with emphasis on sex-specific gene expression. Parasitology, 128: 511522. DOI: $10.1017 / \mathrm{S} 0031182004006213$.

Hotez PJ, Ottesen E, Fenwick A, Molyneux D. 2006. The neglected tropical diseases: the 
ancient afflictions of stigma and poverty and the prospects for their control and elimination. Advance Experimental Biology Medicine, 582: 22-33. DOI: 10.1007/0-387-33026-7_3.

Hussein AS, Kichenin K, Selkirk ME. 2002. Suppression of secreted acetylcholinesterase expression in Nippostrongylus brasiliensis by RNA interference. Molecular Biochemistry Parasitology, 122: 91-94. DOI: 10.1016/s0166-6851(02)00068-3

Inoue N, Otsu K, Ferraro DM, Donelson JE. 2002. Tetracycline-regulated RNA interference in Trypanosoma congolense. Molecular Biochemistry Parasitology, 120: 309-313. DOI: 10.1111/j.1462-5822.2004.00399.x

Islam MK, Miyoshi T, Yamada M, Tsuji N. 2005. Pyrophosphatase of the roundworm Ascaris suum plays an essential role in the worm's molting and development. Infectious Immunology, 73: 1995-2004. Doi: 10.1128/iai.73.4.1995-2004.2005.

Issa Z, Grant WN, Stasiuk S, Shoemaker CB. 2005. Development of methods for RNA interference in the sheep gastrointestinal parasite, Trichostrongylus colubriformis. International Journal of Parasitology, 35: $935-940 . \quad$ DOI: 10.1016/j.ijpara.2005.06.001

Kang S, Hong YS. 2008. RNA $i$ in infectious tropical diseases. Korean Journal of Parasitology, 46(1): 1-15. DOI: https: 10.3347/kjp.2008.46.1.1

Kargbo A, Fatty LKM, Mendy A, Yahaya J, Dibba L. 2020. Biotechnology as a Change Agent for National Development: Review in The Gambia International Journal of Sciences: Basic and Applied Research, 49(2):43-55. DOI: http://gssrr.org/index.php?journal=Journ alOfBasicAndApplied

Kennerdell JR, Carthew RW. 1998. Use of dsRNA-mediated genetic interference to demostrate that frizzled and frozzled 2 act in the wingless pathway. Cell, 95: 10171026. DOI: $10.1016 / \mathrm{s} 0092-$ 8674(00)81725-0
Kim DH, Behlke MA, Rose SD, Chang MS, Choi S, Rossi JJ. 2005. Synthetic dsRNA Dicer substrates enhance RNA $i$ potency and efficacy. Natural Biotechnology, 23: 222-226. DOI: 10.1038/nbt1051.

Kotze AC, Bagnall NH. 2006. RNA interference in Haemonchus contortus: suppression of beta-tubulin gene expression in L3, L4 and adult worms in vitro. Molecular Biochemistry and Parasitology, 145: 101-110. DOI: 10.1016/j.molbiopara.2005.09.012

Kumar S, Chaudhary K, Foster JM, Novlli JF, Zhang Y, Wang S, Spiro D, Ghedin E, Carlow CK. 2007. Mining predicted essential genes of Brugia malayi for nematode drug targets. PLOS ONE, 2: 1189.

DOI:

10.1371/journal.pone.0001189.

Liolios K, Tavernarakis N, Hugenholtz P, Kyrpides NC. 2006. The genomes on line database (GOLD) v.2: a monitor of genome projects worldwide. Nuclear Acids Reserve, 34: 332-334. DOI: 10.1093/nar/gkj145.

Lustigman S, Zhang J, Liu J, Oksov Y, Hashmi S. 2004. RNA interference targeting cathepsin L and Z-like cysteine proteases of Onchocerca volvulus confirmed their essential function during L3 molting. Molecular Biochemistry Parasitology, 138: $\quad 165-170 . \quad$ DOI: 10.1016/j.molbiopara.2004.08.003

Mamoudou A, Mbakou ALM, Ngungwa V, Sevidzem SL, Zoli AP, Achukwi MD. 2016. Preliminary assessment of bovine trypanosomiasis and its vectors in Santa, Bali and Bafut Sub-Divisions of the, North West Region, Cameroon. Int. J. Biol. Chem., Sci., 10(1): 1-12. DOI : http://dx.doi.org/10.4314/ijbcs.v10i1.1

Marie B, Bacon JP, Blagburn JM. 2000. Double-stranded RNA interference shows that Engrailed controls the synaptic specificity of identified sensory neurons. Current Biology, $\quad 10$ : 289292. DOI: $\quad 10.1016 / \mathrm{s} 0960-$ 9822(00)00361-4.

McVeigh P, McCammick EM, McCusker P, Morphew R M, Mousley A, Abidi A. 
Maule AG. 2014. RNAi dynamics in Juvenile Fasciola spp. Liver flukes reveal the persistence of gene silencing in vitro. PLoS Neglected Tropical Diseases, $\quad 8(9)$ : $3185 . \quad$ DOI: 10.1371/journal.pntd.0003185.

Molyneux DH, Hotez PJ, Fenwick A. 2005. Rapid-impact interventions: how a policy of integrated control for Africa's neglected tropical diseases could benefit the poor. PLoS Medicine, 2(11): 336. DOI: 10.1371/journal.pmed.0020336.

Nayduch D, Aksoy S. 2007. Refractoriness in tsetse flies (Diptera: Glossinidae) may be a matter of timing. Journal of Medical Entomology, 44: 660-665. DOI: $10.1603 / 0022-$

2585(2007)44[660:ritfdg]2.0.co;2.

Ngbolua KN, Rakotoarimanana H, Rafatro H, Ratsimamanga US, Mudogo V, Mpiana PT, Tshibangu DST. 2011. Comparative antimalarial and cytotoxic activities of two Vernonia species: $V$. amygdalina from the Democratic Republic of Congo and $V$. cinerea subsp vialis endemic to Madagascar. Int. J. Biol. Chem, Sci., 5(1): 345- 353.

Ngo H, Tschudi C, Gull K, Ullu E. 1998. Double-stranded RNA induces mRNA degradation in Trypanosoma brucei. PNAS, $\quad$ 95: $14687-14692$. DOI: $10.1073 /$ pnas.95.25.14687.

Novina CD, Sharp PA. 2004. The RNAi revolution. Nature, 430: 161-164. DOI: $10.1038 / 430161 \mathrm{a}$

Ntonifor HN, Ajayi JA. 2007. Studies on the ecology and distribution of some medically important freshwater snail species in Bauchi State, Nigeria. Int. J. Biol. Chem. Sci., 1(2):

121-127.

Olson KE, Higgs S, Gaines PJ, Powers AM, Davis BS, Kamrud KI, Carlson JO, Blair CD, Beaty BJ. 1996. Genetically engineered resistance to dengue-2 virus transmission in mosquitoes. Science, 272: $\quad 884-886$. DOI: $10.1126 /$ science. 272.5263 .884

Osta MA, Christophides GK, Kafatos FC. 2004. Effects of mosquito genes on
Plasmodium development. Science, 303: 2030-2032.

DOI: 10.1126/science.1091789.

Oyibo WA, Agomo C, Olasosu OO, Teslim OO, Sanyaolu AO, Ajuluchuckwu J, Fagbenro-Beyioku, AF, Otigbuo I. 2009. Human African trypanosomes: challenges posed to the human immune system. Int. J. Biol. Chem. Sci., 3(1): 156167.

Pfarr K, Heider U, Hoerauf A. 2006. RNAi mediated silencing of actin expression in adult Litomosoides sigmodontis is specific, persistent and results in a phenotype. International Journal of Parasitology, 36: 661-669. DOI: 10.1016/j.ijpara.2006.01.010

Robinson KA, Beverley SM. 2003. Improvements in transfection efficiency and tests of RNA interference (RNA $i$ ) approaches in the protozoan parasite Leishmania. Molecular Biochemistry Parasitology, 128: 217-228. DOI: 10.1016/s0166-6851(03)00079-3

Saito K, Nishida KM, Mori T, Kawamura Y, Miyoshi K, Nagami T, Siomi H, Siomi MC. 2006. Specific association of Piwi with rasiRNAs derived from retrotransposon and heterochromatic regions in the Drosophila genome. Genes Development, 20: 2214-2222. DOI: 10.1101/gad.1454806

Shi H, Djikeng A, Tschudi C, Ullu E. 2004. Argonaute protein in the early divergent eukaryote Trypanosoma brucei: control of small interfering RNA accumulation and retroposon transcript abundance. Molecular Cell Biology, 24: 420-427. DOI: $10.1017 / \mathrm{s} 1355838200000297$

Shi H, Djikeng A, Mark T, Wirtz E, Tschudi C, Ullu E. 2000. Genetic interference in Trypanosoma brucei by heritable and inducible double-stranded RNA. RNA. 6:1069-1076. DOI: 10.1261/rna.246906.

Shi H, Tschudi C, Ullu E. 2006. An unusual Dicer-like1 protein fuels the RNA interference pathway in Trypanosoma brucei. RNA, 12: 1-10.

Shi H, Tschudi C, Ullu E. 2007. Depletion of newly synthesized Argonaute1 impairs 
the RNA $i$ response in Trypanosoma brucei. RNA, 13: 1132-1139. DOI: 10.1186/1475-2875-5-48

Song JJ, Liu J, Tolia NH, Schneiderman J, Smith SK, Martienssen RA, Hannon GJ, Joshua-Tor L. 2003. The crystal structure of the Argonaute2 PAZ domain revals an RNA binding motif in RNA $i$ effector complexes. Natural Structure in Molecular Biology, 10: 1026-1032. DOI: 10.1038/nsb1016.

Tabara H, Grishok A, Mello CC. 1998. Reverse genetics: RNA $i$ in C. elegans: soaking in the genome sequence. Science, 282: 430431. DOI: $10.1126 /$ science.282.5388.430

Tijsterman M, May RC, Simmer F, Okihara KL, Plasterk RH. 2004. Genes required for systemic RNA interference in Caenorhabditis elegans. Current Biology, 14: 111-116. DOI: 10.1016/j.cub.2003.12.029

Viney ME, Thompson FJ. 2008. Two hypotheses to explain why RNA interference does not work in animal parasitic nematodes. International Journal of Parasitology, 38: 43-47. DOI: 10.1016/j.ijpara.2007.08.006

Visser A, Geldhof P, de Maere V, Knox DP, Vercruysse J, Claerebout E. 2006. Efficacy and specificity of RNA interference in larval lifestages of Ostertagia ostertagi. Parasitology, 31: 17. DOI: 10.1017/S0031182006001004.
Wang Z, Morris JC, Drew ME. 2000. Englund PT. (Inhibition of Trypanosoma brucei gene expression by RNA interference using an integratable vector with opposing T7 promoters. Journal of Biology and Chemistry, 275: 4017440179. DOI: 10.1101/gad.13.24.3191

Winston WM, Molodowitch C, Hunter CP. 2002. Systemic RNA $i$ in $C$. elegans requires the putative transmembrane protein SID-1. Science, 295: 2456-2459. DOI: $10.1126 /$ science. 1068836

Yamagata Y, Nakagawa J. 2006. Control of Chagas disease. Advance Parasitology, 61: $\quad 129-65 . \quad$ DOI: 10.1016/S0065-308X(05)61004-4

Zhang WW, Matlashewski G. 2000. Analysis of antisense and double stranded RNA downregulation of A2 protein expression in Leishmania donovani. Molecular Biochemistry Parasitology, 107: 315319 . DOI: $10.1016 / \mathrm{s} 0166-$ 6851(99)00236-4.

Zongo D, Bagayan M, Tiendrebeogo S, Drabo F, Ouedraogo H, Savadogo B, Bamba I, Yago-vienne F, Zhang Y, Poda JN. 2016. Assessment of schistosomiasis and intestinal helminths following mass drug administration in the Centre and Plateau Central regions of Burkina Faso. Int. J. Biol. Chem. Sci., 10(4): 1525-1533.

DOI: http://dx.doi.org/10.4314/ijbcs.v10i4.6 